\title{
THE INDUCTION OF CALCIUM PHOSPHATE GROWTH ON SURFACE FUNCTIONALIZED CELLULOSE OR CHITIN FIBER
}

\author{
YOSHIYUKI YOKOGAWA ${ }^{1}$, HARI KRISHNA VARMA ${ }^{2}$, KAORI NISHIZAWA ${ }^{1}$, FUKUE \\ NAGATA', TETSUYA KAMEYAMA', 'Bioceramics Laboratory, National Industrial \\ Research Institute of Nagoya, 1-1 Hirate-cho, Kita, Nagoya 462-8510, Japan; ${ }^{2}$ Biomedical \\ Technology Wing, Sree Chitra Tirunal Institute for Medical Science and Technology, \\ Trivandrum 695012, India
}

\begin{abstract}
Calcium phosphate growth on surface functionalized cellulose. or chitin was studied. Cellulose or chitin fibers phosphorylated (then soaked in saturated $\mathrm{Ca}(\mathrm{OH})_{2}$ solution at ambient temperature) or treated with silane coupling reagent were found to stimulate the growth of a calcium phosphate coating on their surfaces after soaking in $1.5 \times \mathrm{SBF}$ solution at $36.5^{\circ} \mathrm{C}$ as little as one day. Surface functionalization such as phosphorylation or treatment of silane coupling reagent has been found to be a useful method for making the fiber surface susceptible to biomimetic deposition of calcium phosphate coating.
\end{abstract}

\section{INTRODUCTION}

Earlier, a study was reported ${ }^{1}$ in which the induction of calcium phosphate growth by introduction of phosphorous-containing groups into the biological fibers (cellulose or chitin) was described. Studies of the induction of calcium phosphate growth on surface-treated substrates are of potential value in the design of biomaterials which more closely resemble the natural tissues. However, the cellulose or chitin /calcium phosphate system has the potential use as a virus filter given the adsorptive properties of calcium phosphate ${ }^{2}$ which may allow the attachment of drugs for future treatment of a serious viral disease. In the previous study ${ }^{1}$, it was found that a technique of phosphorylation and partial hydrolysis of functionalities by $\mathrm{Ca}(\mathrm{OH})_{2}$ treatment to create hydrolysis products in intimate contact with the substrate has been found to be a useful method for creating the favorable local conditions leading to the growth of calcium phosphate on fibers. In this present study, a treatment of silane coupling reagent as well as phosphorylation as a method for creating favorable local conditions leading to the growth of calcium phosphate on fiber was studied.

\section{EXPERIMENTAL}

The experimental procedure for phosphorylation of the fibers using urea/ $\mathrm{H}_{3} \mathrm{PO}_{4}$ method has already been described in an earlier report ${ }^{1}$. Tetraethoxy silane (TEOS) treatment was carried out as follows. Fibers were placed into a flask. Care was taken to make sure that the flask is thoroughly dried. $50 \mathrm{~mL}$ TEOS was added to the flask. The solution was heated at $125^{\circ} \mathrm{C}$ for 1 hour. After cooling the flask. fibers were washed with ethyl alcohol and washed in water to remove any excess alcohol.

Received January 22, 1999; approved April 18, 1999 
0.05-0.1g of samples of $\mathrm{Ca}(\mathrm{OH}) 2$-treated phosphorylated fiber or TEOS treated fibers were placed into plastic screw-top flask to which $10-20 \mathrm{~mL}$ of $1.5 \times \mathrm{SBF}$ solution added. A precise description of $1.5 x \mathrm{SBF}$ solution was published in earlier report ${ }^{3}$. The ion concentrations of SBF solution are almost the same as those of body plasma solutions. The flasks were immersed in a thermostatically controlled $36.5^{\circ} \mathrm{C}$ covered water bath for periods of $1,2.4,6,9,10.17,20$ and 30 days. The $1.5 x$ SBF solution was replaced each day. All samples were dried at $40-60^{\circ} \mathrm{C}$ under vacuum. The morphology of the samples were observed by scanning electron microscopy and EDX analyses were performed. The micro-FTIR spectra were recorded of samples encased in a transparent $\mathrm{KBr}$ matrix and ATR (attenuated Total Reflectance) technique also was applied. Determination of $\mathrm{P}$ content of the fibers was carried out. Each phosphorylated fiber was dissolved in a mixture of water, $\mathrm{H}_{2} \mathrm{O}_{2}$ and $\mathrm{H}_{2} \mathrm{SO}_{4}$ and then diluted to apply to the ICP emission spectroscopic analysis.

\section{RESULTS AND DISCUSSION}

Morphologically, the appearance of the phosphorylated or TEOS treated fibers under the electron microscope appeared unchanged from that of as-received fibers. EDX spectrum of the TEOS treated fiber showed strong silicon signal was observed. The amount of silicon estimated by ICP analysis was found to be at $0.03-0.05$ mass $\%$ of the fiber and lower than the P content of phosphorylated cellulose (4.06 mass\%) and chitin (2.95 mass\%). Micro-FTIR spectra of unphosphorylated cellulose (Fig.1(a)) and phosphorylated cellulose (Fig.1(b)) confirmed that chemical modification of the fibers had taken place. Broadening of the typical cellulose band was observed at $1000 \mathrm{~cm}^{-1}$ which correspond the P-O stretching band from $\mathrm{PO}_{4}$ groups chemically bonded to cellulose fibers. The chitin fibers phosphorylated indicated the similar phenomena. That of cellulose after TEOS treatment (Fig.1(c)) showed the peaks at 470 and $801 \mathrm{~cm}^{-1}$ due to the Si-O-C bond were observed. There is considerable reduction in the intensity of hydroxyl peaks in the region $3600-2500 \mathrm{~cm}^{-1}$ due to the reaction between the $\mathrm{OH}$ group of cellulose and the $\mathrm{SiOC}_{2} \mathrm{H}$ s.

Soaking of the $\mathrm{Ca}(\mathrm{OH})_{2}$-treated phosphorylated fibers in $1.5 \mathrm{xSBF}$ solution was found to lead to the deposition of a calcium phosphate layer. It is believed that the thin coatings of calcium phosphate on the fibers produced by partial hydrolysis of the fiber- $\mathrm{PO}_{4}$ functionalities by soaking in saturated $\mathrm{Ca}(\mathrm{OH})_{2}$ solution act as a nucleation layer upon which the calcium phosphate can grow from $1.5 \mathrm{xSBF}$ solution. In general, the growth of calcium phosphate from $1.5 \mathrm{xSBF}$ solution begins immediately after 1 day of soaking. This suggests that clusters have partially dissolved upon introduction of the fibers into the $1.5 \mathrm{xSBF}$ solution. The growth of calcium phosphate layer after soaking for 1-6 days appears to proceed by nucleation on the existing coating in the form of circular flakes which then grow in number and size on the surface. After soaking for 9-17 days, a thicker coating was observed. 


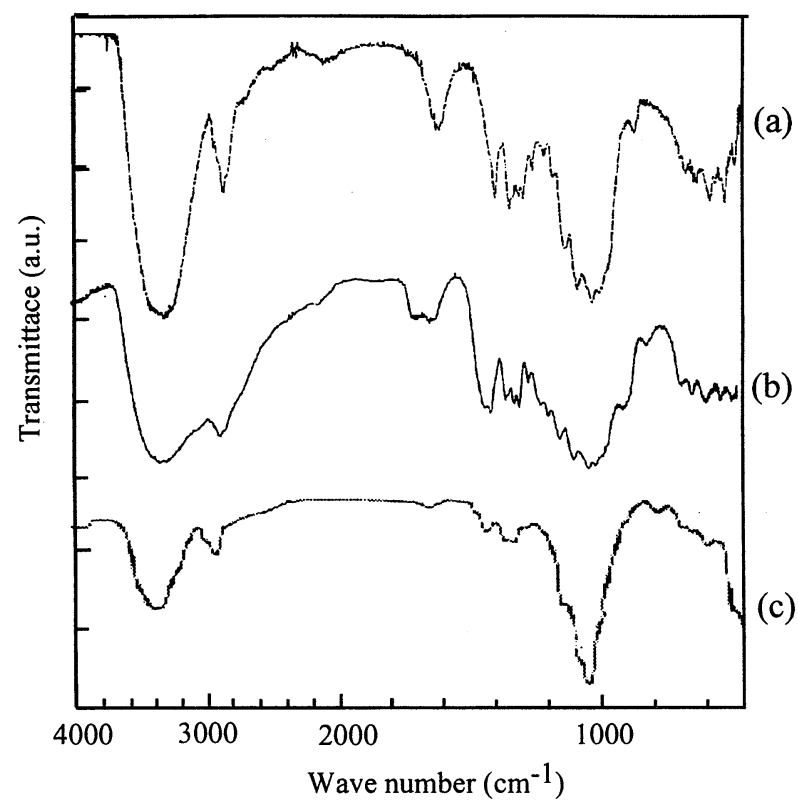

FIGURE 1 Micro-FTIR spectrum of cellulose sample (a) before. (b) after phosphorylation, and (c) after TEOS treatment.
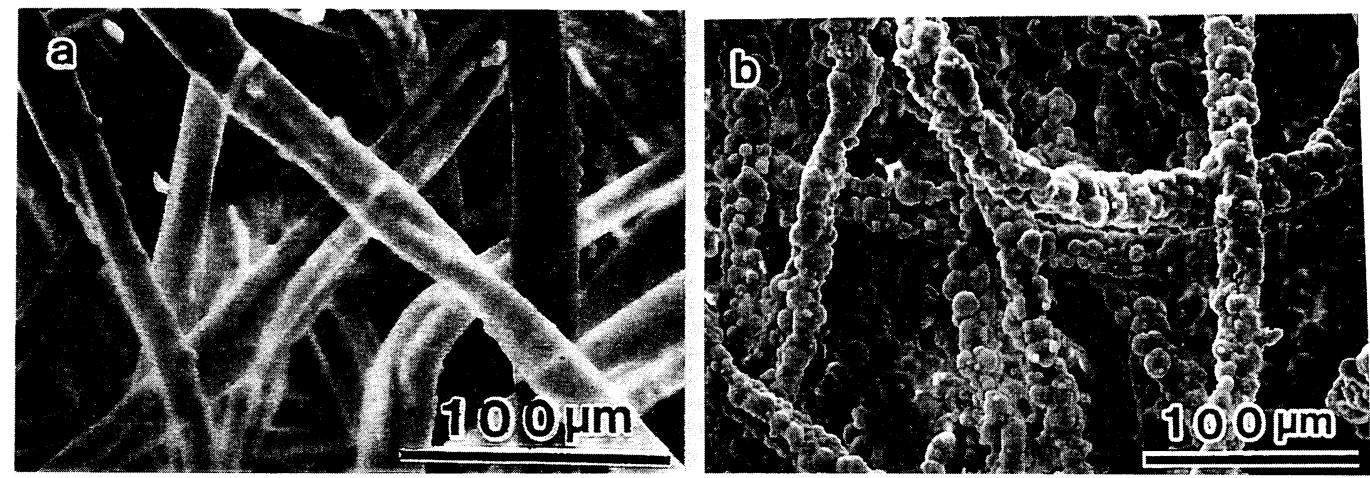

FIGURE 2 Scanning electron micrograph of sample of (a) $\mathrm{Ca}(\mathrm{OH})_{2}$-treated phosphorylated cellulose fibers soaked in $1.5 \times \mathrm{SBF}$ solution at $36.5^{\circ} \mathrm{C}$ for 17 days, and (b) TEOS treated cellulose fiber in $1.5 \mathrm{xSBF}$ solution for 30 days.

Nucleation of an initial calcium phosphate layer was started to occur all over the fiber surface within 3 days in case of TEOS treated fibers. Later growth of secondary particles in the form of spherical cluster has occurred over this initial layer. The secondary growth is a random phenomena and as the aging time increases, the fiber became completely covered with such nodular clusters of calcium phosphate. The each spherical particles are appeared as porous. The surface became completely covered by thick coating after 20 days and the phase is identified as carbonated substituted apatite. The EDX 
measured $\mathrm{Ca} / \mathrm{P}$ ratio for the calcium phosphate coatings on $\mathrm{Ca}(\mathrm{OH})_{2}$-treated phosphorylated fibers or TEOS treated fibers as a function of soaking time in $1.5 \times \mathrm{SBF}$ solution show the similar behavior shown in Fig.3. They are, in general, scattered and appear to increase with an increase in the soaking time but appear to stabilize for thick coatings (formed at soaking times $>9$ days) at a value of around 1.60, suggesting Ca-deficient apatite. It is evident that carbonate is present to a noticeable extent in the surface functionalized fibers soaked in $1.5 \mathrm{xSBF}$ as demonstrated by the broad peak at around $1400 \mathrm{~cm}^{-1}$ in FTIR spectrum of the coating material grown on the fibers.

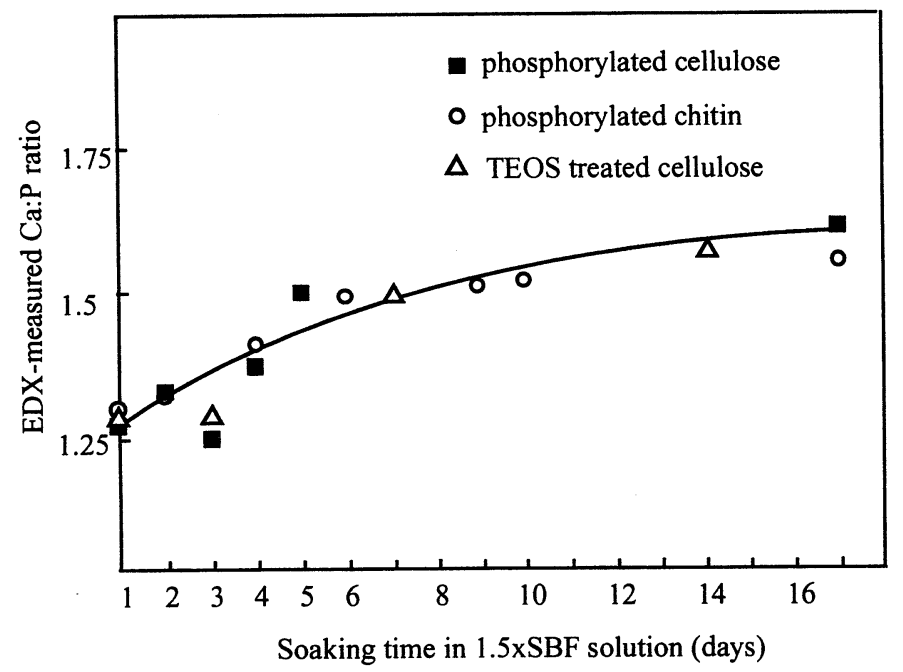

FIGURE 3 EDX-measured $\mathrm{Ca} / \mathrm{P}$ ratios for the calcium phosphate coatings as a function of soaking time in SBF solution.

\section{CONCLUSION}

Cellulose or chitin fibers phosphorylated (then soaked in saturated $\mathrm{Ca}(\mathrm{OH})_{2}$ solution at ambient temperature) or treated with silane coupling reagent were found to stimulate the growth of a calcium phosphate coating on their surfaces after soaking in $1.5 \times \mathrm{SBF}$ solution at $36.5^{\circ} \mathrm{C}$ as little as one day. This study into organic polymer substrates designed to enhance the growth of calcium phosphate on their surface demonstrates that surface functionalization such as phosphorylation or TEOS treatment has been found to be a useful method for making the fiber surface susceptible to biomimetic deposition of calcium phosphate coating.

\section{REFERENCES}

1. Y. Yokogawa, M.Toriyama, Y.Kawamoto, T.Suzuki, K.Nishizawa, F.Nagata, T.Kameyama, and M.R.Mucalo, and J.P.Reyes, Phosphorous Res. Bull., 6, 59 (1996)

2. S.Tsuru, N.Sinomiya, Y.Katsura, Y.Uwabe, M.Noritake, and M.Rokutanada, Bio-Med.Mater.Eng., 1 , 143(1991)

3. M. R. Mucalo, Y. Yokogawa, M. Toriyama, T. Suzuki, Y. Kawamoto, F. Nagata, and K. Nishizawa, J. Mater. Sci. Mater. Med., 6, 597(1995) 